\title{
The Day after Tomorrow: How Should We Address Health System Organization to Treat Cancer Patients after the Peak of the COVID-19 Epidemic?
}

\author{
Jean-Jacques Tuech ${ }^{a, b} \quad$ Alice Gangloff ${ }^{c}$ Frederic Di Fiore ${ }^{b, c}$ \\ Ahmed Benyoucef $^{d}$ Pierre Michel ${ }^{b, c}$ Lilian Schwarz ${ }^{a, b}$

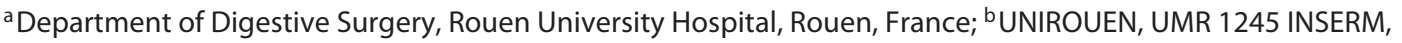 \\ Rouen University Hospital, Department of Genomic and Personalized Medicine in Cancer and Neurological \\ Disorders, Normandie University, Rouen, France; ' ${ }^{C}$ epartment of Digestive Oncology, Rouen University Hospital, \\ Rouen, France; ${ }^{d}$ Radiotherapy Department, Centre de Lutte Contre le Cancer Henri-Bequerel, Rouen, France
}

\section{Keywords}

Pandemic · COVID-19 outbreak · Cancer · Oncology · Health care systems

\begin{abstract}
On March 11, 2020, the WHO director general declared COVID-19 a pandemic. This pandemic evolves in successive phases, i.e., phase 1 (the start phase), phase 2 ("the storm"), and phase 3 (the recession). To date, oncology and surgery groups have only given instructions for addressing phases 1 and 2. To prevent excess cancer mortality, health care systems (HCS) need to be restructured. Our aim is to detail the specificities of each epidemic phase and discuss several methods of organization to optimize cancer patient flow during the COVID-19 pandemic, particularly during phase 3 . Hospitals must be reorganized in order to create a cancer hub that is free of infection, allowing for the safe treatment of patients. Hospital structures are different, but all allow for the creation of virus-free areas. Screening programs are critical and need to be applied to all people entering the virus-free zone, including health care workers. Some reorganization
\end{abstract}

proposals are internal to a hospital, while others require interhospital collaboration. The heterogeneity and complexity of HCS will make interhospital management difficult. The ministry of health has an important role in managing the cancer crisis. Cancer management should be declared a priority. Oncological and surgical societies must coordinate their efforts to facilitate this prioritization. The anticipation of oncological management during phase 3 of the pandemic is necessary because it requires a complete readjustment of HCS. This adaptation should allow for the continuation of cancer care to prevent excess cancer mortality, as the virus will still be present for a currently undetermined period of time.

(c) 2020 S. Karger AG, Basel

\section{Introduction}

In response to the COVID-19 epidemic, dramatic heath system reorganization has been necessary to treat the first wave of infected patients. In this context, cancer patient management has been significantly impacted, from diagnosis to follow-up $[1,2]$. karger@karger.com

www.karger.com/ocl

Karger ${ }^{\prime}=$ (c) 2020 S. Karger AG, Base

Department of Dige

Surgery, Hopital Charles Nicolle

FR-76031 Rouen (France)

jean-jacques.tuech@chu-rouen.fr 
Fig. 1. From left to right, the blue curve shows the increase and decrease in the number of infected cases during the 3 phases of the outbreak. During phases 1 and 2, the risk is a shortage of intensive care beds, and the goal of the health authorities is to flatten the growing curve of the virus (curve in blue dots). During this time, cancer patients cannot be treated, which is represented by a decrease in the green curve during the first 2 phases. A waiting list of cancer patients develops during this period. In the absence of anticipatory measures, the capacity of the oncological system will be overwhelmed by the massive influx of cancer patients from the waiting list but also from the normal flow. The objective is to flatten the curve by taking adaptive measures with the aim of not altering the prognosis of cancer patients. The possible second wave of the epidemic has not been represented on these curves. The area under the curve for the light green and dark green curves should be considered as equal.

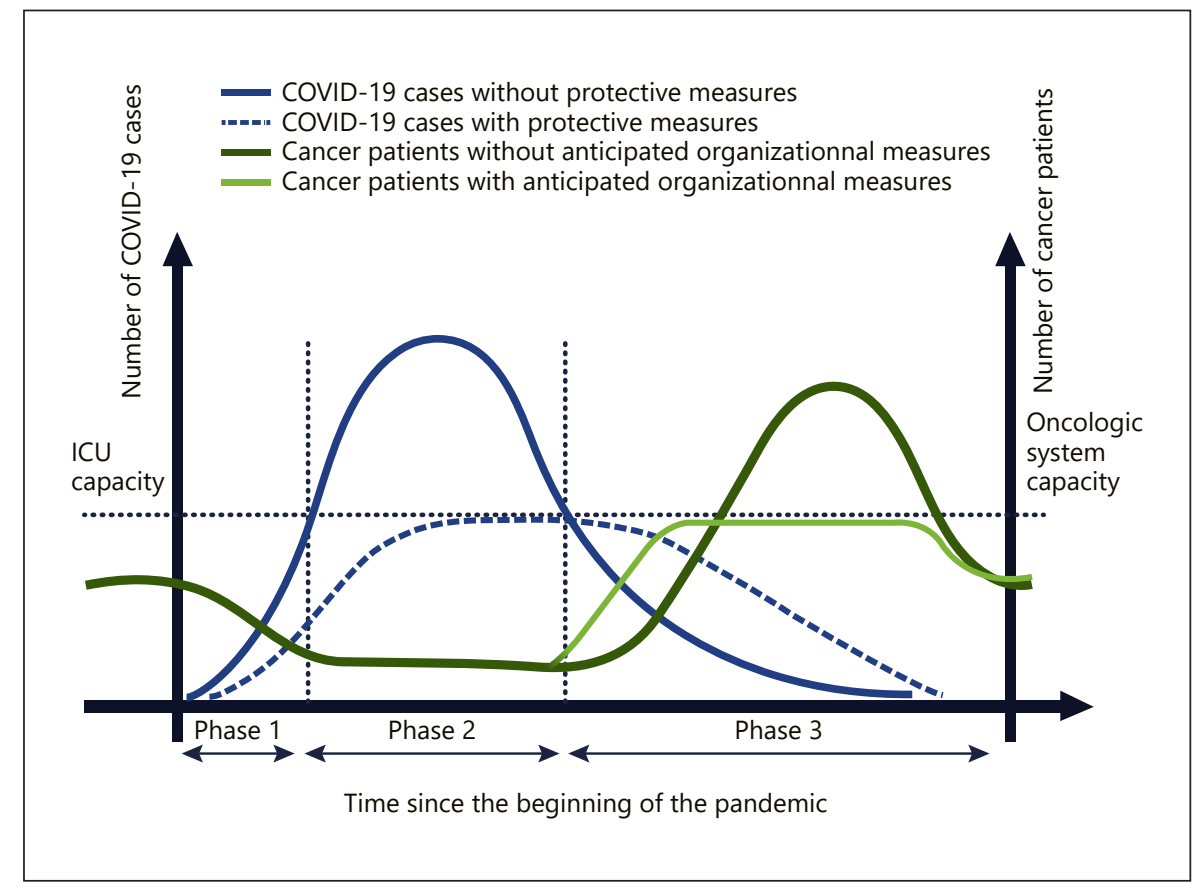

Although the epidemic is still active, some countries have now attempted to address the peak of the epidemic, and the management of patients with cancer in this new context remains questionable. Each year in France, 181,341 people die of cancer and 412,952 people learn that they have the disease [3]. To prevent excess cancer mortality [4], we believe that healthcare systems need to be completely reorganized to provide the most appropriate network for patients and caregivers. Change is the basic law of nature, but "it is not the strongest species that survive, nor the most intelligent, but the ones most responsive to change" (Charles Darwin).

We do not have enough beds, equipment and staff to care for a surge of patients in a widespread, rapidly escalating outbreak. The American College of Surgeons (ACS) released the "COVID-19 recommendations" for the management of elective surgical procedures and recommended minimization, postponement, or cancellation of electively scheduled surgeries and invasive procedures [5]. This work by the ACS underlines that the response to the crisis must be adapted to the evolution and known phase of the pandemic. To avoid being swamped with sick patients, public health authorities aim to flatten the spread curve of the virus (Fig. 1). Flattening the curve will probably save lives. However, lockdown is likely to prolong the duration of the epidemic by delaying the time taken to reach herd immunity in the absence of an effective vac- cination. At this time, we are at the beginning of the crisis, but we can already anticipate the needs of our medical organizations so that they can be set up gradually as the pandemic evolves. Thus far, most oncology and surgery groups [6-10] have only given instructions or recommendations for the phase before and during the peak of viral contamination.

We can determine 3 phases for this pandemic (Fig. 1). Phase 1 is the start phase. Phase 2 is "the storm," with a number of cases that doubles very quickly. Phase 3 is the recession phase, which can be followed by a return to normal or by a new cycle (second wave).

We detail here the specificities of each epidemic phase from an oncological point of view and discuss several organizations to optimize the flow of cancer patients during the COVID-19 pandemic.

\section{Health System Proposals According to the Pandemic Phases}

This pandemic is evolving by cluster, thus rendering adaptation nonstandardized within the same country. Indeed, some regions may be in phase 1 , while others will be in phase 2 or in phase 3 . As a result, adaptation will have to be regional rather than national, at least during phases 1 and 2 . 
Several recommendations $[6,7,11,8-10]$ have been published on oncologic management during the pandemic; however, it is unclear when these recommendations should be applied. Phases 1 and 2 are the most impressive but have a relatively short duration, and the oncological impact will be moderate for individual care. Phases 1 and 2 will generate a significant number of patients to be treated during phase 3 . Phase 3 will last for a long time, and the oncology care system could be overwhelmed (Fig. 1).

In light of this major problem, adaptation during phase 3 is in fact the real challenge of oncology, as we will be confronted with individuals who expect personalized care but for whom the response will need to be integrated into an overall structural organization focused on virus risk prevention.

\section{During Phase 1 (Calm before the Storm)}

Phase 1 is already behind us. However, the adaptations are important to analyze because a second wave is possible.

During this phase, we must prepare the management of phase 2 to make it as nonlethal as possible. The aim was to postpone the peak of the epidemic to have enough beds in the intensive care units (ICU). Activities must be reduced to dispatch enough healthcare workers (HCW) to the COVID ward. It was asked to postpone or cancel elective and nonurgent procedures. The emergency and resuscitation departments must reorganize the hospital around COVID-19 management. The mental load induced by this work is so important that it is necessary to give them time to make this reorganization possible.

For cancer patients, diagnosis and treatment should be tailored to the best available resources. As patients with cancer are more likely to develop severe forms than are individuals without cancer [12-15], some groups have proposed [6, 7, 11, 8-10] postponement of nonurgent treatment in favor of a chemotherapy regimen that induces less neutropenia. Radiotherapy treatment could also be postponed because patients have to be treated every day, which increases the risk of contamination when going to and from the hospital. To minimize this risk of contamination, some have proposed adapting dose modulation [16]. What is certain is that when radiotherapy treatment has started it should not be interrupted $[9,17]$.

There are points in common in the care of cancer patients, i.e., (1) prevent undernutrition; (2) avoid significant deterioration of the immune system by aggressive treatments; (3) avoid hospitalizations, visits, and hospital stays that would favor contamination with the virus; (4) favor a therapeutic sequence that does not impose a strict surgical timing that cannot possibly be achieved; (5) discuss each case in a multidisciplinary meeting on a regular basis to adapt the individual strategy to the duration of phases 1 and 2; and (6) offer the availability of psychological support to patients who have to face cancer and the fear of infection.

All unnecessary consultations should be postponed and replaced by dematerialized consultations to reduce the risk of cross-infection. If the consultation is essential, the relevant CDC recommendation [18] must be strictly observed. Patients with cancer frequently need hospitalization, and it seems that the hospitalization sectors must be fitted out in individual rooms to limit cross-infections with other patients; individual rooms also facilitate the management of isolation. It seems important to inform the patient and their family that visits are not allowed. Even if the treatment times are longer than usual due to the precautions taken to avoid cross-infection, it seems advisable to set up a dematerialized communication system with a member of the family.

Among the problems that this crisis raises, one of them is the elevated risk of HCW of exposure to the virus. It is mandatory to ensure the safety of HCW for their own wellbeing and to safeguard continuous patient care [19-21].

\section{During Phase 2 (the Storm)}

During this phase, all efforts are focused on ICU management to save the lives of COVID-19 patients. It seems unrealistic to be able to treat a patient with cancer during this phase while we are building makeshift hospitals on the streets of our cities. The adaptations described for phase 1 can be applied during phase 2 if conditions are favorable. If a patient has received a treatment that can induce neutropenia, regular blood tests should monitor this parameter, and hematopoietic colony-stimulating factor should be used if necessary.

\section{During Phase 3}

The duration of phase 3 is unpredictable, but it is likely to last several weeks or several months. This duration, by itself, justifies modifications to our health care system (HCS).

During this phase, we will have to manage infected patients, immunized patients, and patients who have not been infected by the virus. The latter can be infected but asymptomatic.

Infected patients must be cared for by a dedicated infection department and isolated from other citizens. Oncologic treatments must be interrupted, except, as we 
Table 1. Symptom checklist and history exposure for COVID-19

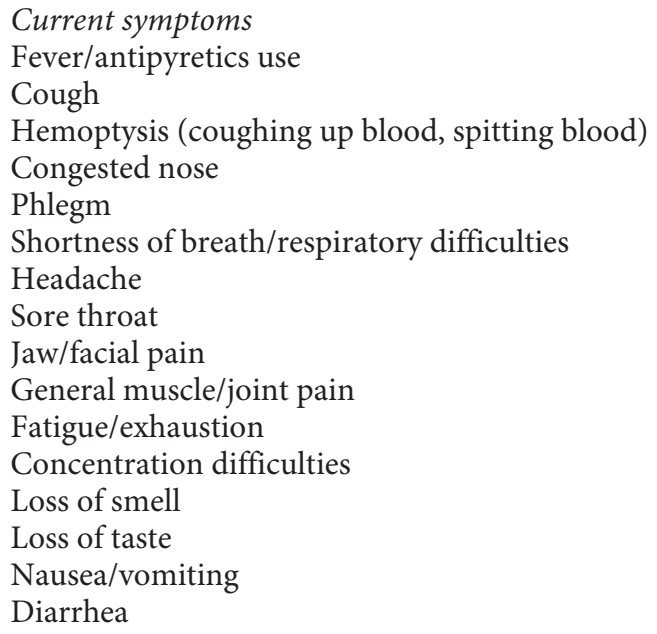

Clinicians should use their judgment to determine whether a patient has signs or symptoms consistent with COVID-19 and whether the patient should be tested. Most patients with confirmed COVID-19 have developed fever and/or symptoms of acute respiratory illness (e.g., cough), but some infected patients may have other symptoms as well, as listed.

mentioned above, for radiation therapy that has already started and for hormone therapy. If a patient has received a treatment that can induce neutropenia, regular blood tests should monitor this parameter, and hematopoietic colony-stimulating factor should be used if necessary. There is currently no evidence on the value of prophylactic use of these treatments during a pandemic.

Immunized patients should be treated as they would have been before the pandemic. Large-scale deployment of serological testing is underway internationally. If immunization is documented, this information should be recorded in the patient's medical record because a new SARS-CoV-2 infection seems unlikely [22].

The necessary adaptive measures that we have to set up must take into account the coexistence of these 3 groups of patients. We must be particularly vigilant for uninfected patients and those with multiple comorbidities who are more likely to develop severe forms. The main objective of phase 3 will be to continue to control the epidemic while managing the majority of asymptomatic or nonimmune patients. All efforts to limit mortality from COVID-19 should not lead to excess mortality due to acute or chronic pathologies for the rest of the population (COVID free) [4].

\section{Education and Information for Cancer Patients}

Today, everyone is aware of the risk of infection. Cancer patients should be informed that they are more likely to develop severe forms. Information must be delivered on hand washing, social distancing measures, the symptoms and signs of infection, the risks of multiple travel trips, and the need to inform healthcare personnel of any new symptom that may give rise to a fear of infection.

\section{Screening Program before a Medical Consultation or Hospitalization}

The purpose of this screening is to prevent an infected patient from coming to the oncology clinic [23]. A phone call should be made the day before the appointment using a screening checklist (listing: travel, contact with infected patients, symptoms related to a possible infection, temperature, and antipyretics) (Table 1). There are, to date, no specific guidelines regarding the use of masks in cancer patients. However, these vulnerable patients should be protected as much as possible, and the wearing of masks may seem useful in this particular period.

A final check will be carried out before admission in a dedicated room. Patients have their temperature checked twice in the waiting area and inside the clinics, with at 
least a 10-min interval between the 2 checks [24]. In case of doubt about a possible infection, the patient is transferred to the dedicated COVID-19-positive stream, and management will be performed according to the recommendations $[19,25]$. In all cases, these recommendations must be observed due to the large proportion (up to $33 \%$ ) of asymptomatic forms [26, 27].

All people entering the clinic, including HCW, must undergo the same screening method.

The aim of such screening is not only to prevent SARSCOV-2 from entering a COVID-free hospital but also to reassure patients and convince them to accept adequate oncological treatment.

Today, a question arises: should or can we go further in the detection of asymptomatic cases?

In symptomatic patients, the sensitivity of computed tomography (CT) and RT-PCR is 98 and $71 \%$, respectively [28]. There are currently very little data on the results of these examinations in asymptomatic patients. In a recent study, 337 asymptomatic patients were detected by RT-PCR, and none of them were found to be positive [29]. Zhang et al. [30] reported the CT characteristics of SARS-CoV-2 pneumonia in 16 asymptomatic patients. Thus far, we do not have useful examinations to propose this intensification of screening. In addition, this route is costly and resource intensive and adds pressure to a hospital system already under strain.

\section{Outpatient Consultation}

Outpatient visits to the clinic should be reduced without jeopardizing oncologic patient care. All nonessential consultations must be replaced by dematerialized consultations. Reducing the number of consultations has 2 aims: to reduce the risk of infection of nonimmunized patients and staff and to anticipate the foreseeable decline in consultation possibilities. This decrease is due to 2 factors. The first factor is that consultations will probably be longer because patients' stress increases, since they must face cancer as well as the infection risk. The second factor is that consultations must be scheduled with long intervals between successive appointments to prevent crowding in waiting rooms.

Contacts with patients must be limited. If a clinical examination is essential, then it must be carried out with gloved hands. HCW must change their gloves immediately after being in contact with patients, body fluids, or contaminated materials. Quick-drying water-alcohol gel must be used for hands.

Cancer survivor patients are good candidates for telemedicine follow-up [31]; however, the rescheduling of this visit should be planned to prevent the patient from being lost to follow-up.

\section{Hospitalized Patients}

Cancer patients are frequently hospitalized either for urgent symptoms or for medical or surgical treatment. As we mentioned above, all of these hospitalizations require a strict triage to keep the negative COVID stream free of virus infection [32].

Before hospitalization for elective surgery or immunosuppressive chemotherapy, it may be necessary for and very important that a patient be isolated at home for a certain period (such as the 14-day quarantine period recommended by the WHO) to reduce the risk of being infected.

It seems optimal for the hospitalization ward to be sectioned in individual rooms during the epidemic period to forestall cross-infections with other patients. Individual rooms also facilitate isolation management if necessary. It seems important to inform the patient and their family that visits should be prohibited. These limitations are intended to protect the patient, other patients and the staff. This limitation could generate a higher level of stress and anxiety than before the pandemic, and this situation must be anticipated. It is advisable to set up an electronic individual communication system, in addition to the telephone, to create virtual proximity to the patient and his/ her family. Information on the patient's state of health may also be communicated to a family member if the patient agrees with this or requests it on a regular basis.

Before starting treatment, some asymptomatic patients may be isolated and quarantined if, after triage, doubt persists, such as contact with a possibly infected person. Indeed, screening tests have a low sensitivity and today do not eliminate a latent or early infection. Differential diagnoses, especially the adverse effects of medical treatment, surgical complications, and other causes of infection, should be eliminated.

This COVID-negative hospitalization area must be seen by caregivers and patients as a sanctuary, and rigorous application of infection prevention and control measures in health care facilities is mandatory $[33,34]$.

\section{HCW in the Oncology Department}

At the beginning of phase 3 , it might be possible that the workforce will be affected by measures taken by the state or the government (confinement, lifting of confinement, closing of schools, etc.), and the activity will have to be initially adapted to gradually return to normal. However, at the start, work flexibility should be facilitated 
to reduce stress for HCW and to maintain a sufficient level of staff $[35,36]$.

Staff must strictly apply the measures recommended by the CDC in the hospital. In their daily lives, outside of hospitals, HCW have to follow the recommendations found on the WHO and CDC websites for the general population [37]. All staff must receive full training on how to behave and how to protect themselves and others in and outside of the hospital.

Adequate personal protective equipment must be available, but their use must be precise and without waste because of the risk of shortage.

Meetings are risky situations for infection; all unnecessary meetings must be canceled.

On the other hand, multidisciplinary meetings must be maintained because they allow the right treatment for the right patient to be determined in a personalized manner. These meetings must be held in small groups (team segmentation practice) to minimize the risk of infection and secondarily enlarged according to the prevalence of virus infection.

As Ngoi et al. [38] believe, staff morale was expected to be affected during the crisis by the workload of team segregation, the cancellation of leave, and the enforcement of social distancing. Strategies to boost morale need to be developed.

\section{Oncologic Treatment}

Many medical and surgical societies or groups have published emergency guidelines for the management of cancer during the COVID-19 pandemic $[6,7,11,8-10]$. As we mentioned above, these recommendations are mainly intended for management during phases 1 and 2. These treatment changes have been useful as long as optimal treatments cannot be offered to patients. These treatment modifications, which are mandatory during the crisis, have resulted in a large waiting list, particularly for patients awaiting surgery. The lists of patients awaiting surgical intervention will be the longest to resorb because the surgical capacities will return to normal very gradually. This return to normal for surgery will depend on the number of anesthesia teams available. These teams will probably be very tired after the management of phase 2 and still engaged in ICU management during phase 3 , which seems to start with a slowly decreasing tray. Another point to consider is that a significant number of patients whose surgeries have been postponed have received treatment with chemotherapy on hold. The timing of the rescheduled surgery will depend on the end of the chemotherapy, which complicates the management of reprogramming. The medical records of all patients should be analyzed to determine the date and specific details of the last treatments and the date of the last imaging workup. Most patients will need to be seen for consultation to check their general and nutritional status and to assess whether the cancer has progressed. Fragile patients may have been weakened by confinement, a lack of physical exercise, and the development of cancer. These weaknesses must be addressed before these patients start aggressive treatment.

The adaptive guidelines for managing the crisis will be useful during the start of phase 3 and will allow for the management of the waiting lists without altering the patients' prognosis. However, as soon as possible, optimal treatments should be offered to patients in the most secure environment possible. In the field of surgery, after the resumption of activities, there will be a short favorable period, which will partly allow for the treatment of the accumulated cases. This will be possible because during phases 1 and 2 there have been no diagnoses of new cases. The duration of this favorable period will be linked to the duration of the diagnosis and the duration of the cancer staging workup; additionally, some cases will need neoadjuvant treatment before surgery.

\section{Hospital Reorganization}

Most hospitals treat infected patients in dedicated units, with a proximity between the COVID-positive units and the COVID-negative units and departments used by both. To offer aggressive treatments to patients weakened by cancer or surgery in the safest possible environment, it seems necessary to reorganize our hospitals. The goal is to create a cancer hub that is uncontaminated by the infection. In many countries, hospital-based cancer treatments are integrated into general hospitals and contribute to a clean cancer hub, which could be complicated by the mandatory testing of anyone who accesses the hospital. We are at war against this pandemic, and we must not underestimate the adaptability of our systems, as in real wars. General Patton used to say during WWII, "Don't tell people how to do things; tell them what to do and let them surprise you with the results." We could have applied this principle, but we preferred to consider the possible organizations according to the most common architecture in hospitals. The different proposals can be combined to adapt to local constraints, and the ingenuity of each will do the rest. 
Fig. 2. Possible reorganization of monobloc hospitals. Areas in blue are virus-free zones, areas in yellow are contaminated viral zones, and areas in green are uncertain viral status zones. All patients use the green road to the triage area. After screening, the patient is redirected according to the presumed viral state to the yellow or blue area. Dedicated elevators are identified and used by dedicated patients. The blue elevator cannot stop on the yellow floors, and the yellow elevator cannot go up to the blue floors. Green floors are shared common areas (technical units, radiology departments, etc.). If a second wave of infection hits the country, the COVID-negative floors will gradually be converted into COVID-positive floors. The yellow and blue arrows show the possible evolution in the case of a second wave.

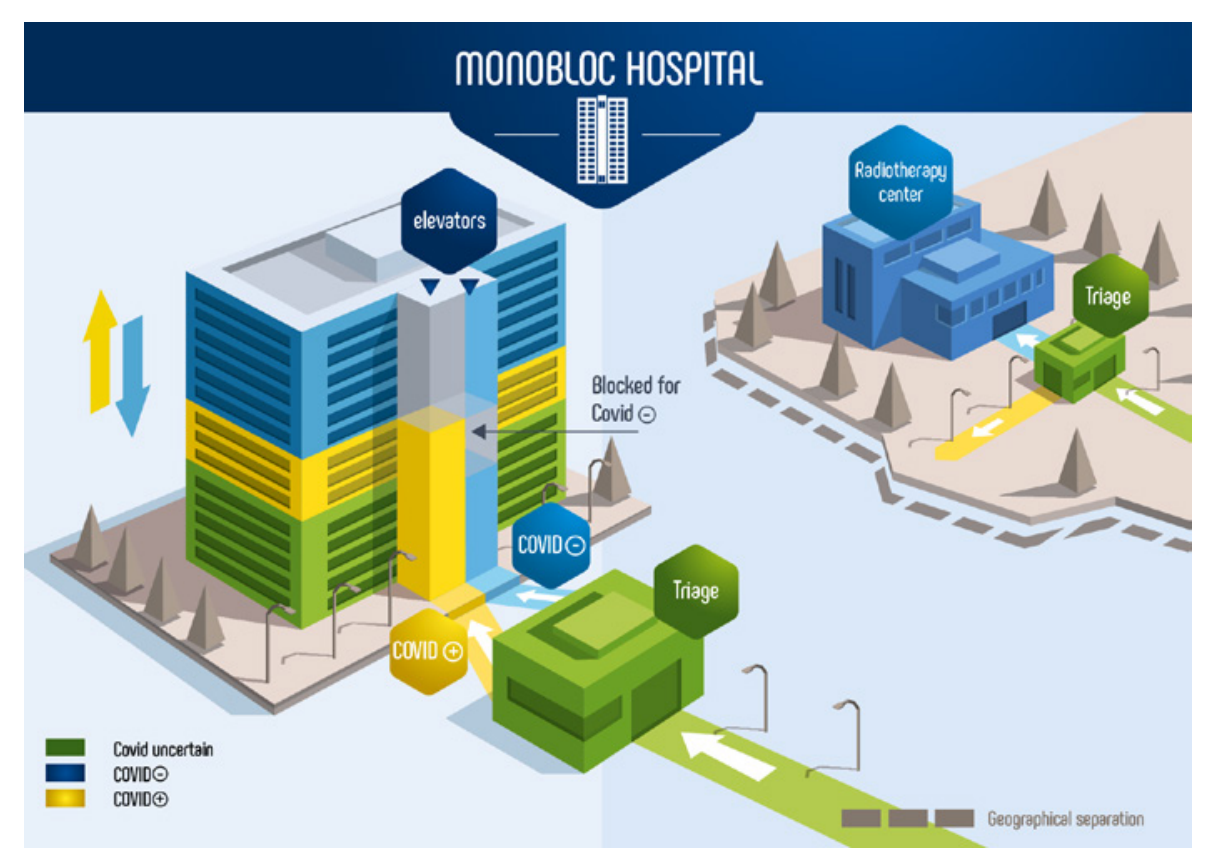

The radiotherapy center is sanctuarized as a negative COVID center, and COVID-negative centers have a triage zone. Various proposals could be made (hospital reorganization [HR] 2-6 as follows), but the organization adapted to the majority of hospitals (HR1) is shown in Figure 2.

\section{HR1: Monobloc Hospital or Podium with 1 Tower}

In this kind of building, several floors will serve COVID-negative patients, and others will serve COVID-positive patients. Several floors will be shared with a mix of COVID-positive patients and COVID patients. These last floors will accommodate the radiology department, an ICU, a pharmacy, a food supply department, and all technical departments. An ICU should be created in the COVID-negative area, as was done during the ICU crisis. In the mixed zone, drastic precautions must be applied to avoid cross-infection; elevators dedicated to each group must be clearly identified.

\section{HR2: Hospital Organized on Campus with Individual Buildings}

One or several buildings will be dedicated to COVIDnegative patients and other to COVID-positive patients. The COVID-negative hospital should ideally include radiology and biology departments, an ICU, a pharmacy, and a food supply department. The radiology department must offer diagnostic examinations, biopsies, drainage, and embolizations, and we should not consider failure to rescue as an option.

Treatment of Cancer Patients after the Peak of the COVID-19 Pandemic

\section{HR3: Monobloc Hospital with a Podium and 2 or More Towers}

This kind of architecture allows for the creation of a COVID-negative area in one tower and a COVID-positive area in another tower. The distribution of the clinical and technical departments will be complete, as in HR1.

\section{HR4}

In this proposal, we considered that the prevalence of the infection would remain very high for many weeks, justifying that the most important hospital in the region is entirely dedicated to the treatment of COVID-positive patients. In such scenarios, another structure (public or private hospital) will be dedicated to the treatment of COVID-negative patients. As in HR1, this COVID-negative hospital must have all departments and full competence to treat all kinds of complications and prevent failure-torescue situations.

\section{HR5}

In this proposal, we considered that the prevalence of the infection would be low, and the most important hospital in the region will be entirely dedicated to the treatment of COVID-negative patients. In such scenarios, another structure (public or private hospital) will be dedicated to the treatment of COVID-positive patients. 
HR6

In this scenario, we considered that, in the same country, cluster areas with a high prevalence of infection and areas with a low prevalence could coexist. In this situation, consideration could be given to the transfer of patients in need of a treatment from regions of a high prevalence to regions of a low prevalence. After being quarantined in the low-prevalence region, these patients could be managed with a high safety level.

\section{The Role of Governments and Ministries of Health}

While HR1-3 depend only on the internal organization of one hospital, HR4-6 are more complicated to implement. Indeed, these last 3 reorganizations require collaboration between hospitals, which often depends on 2 different funding systems. These hospitals are not used to working together on often-diverging interests. At one end are the public hospitals, which will have to take care of patients who test positive for COVID; at the other end is the for-profit hospital, which will take care of profitable pathologies in order to make money. In between, there is a wide variety of hospitals with intermediate goals, but all of them are working with a silo mentality. The silo mentality is most frequently used in the context of a single organization; however, this concept can also be applied to the healthcare industry. The context of the vast, complex, and multiplayer landscape of the HCS can make interhospital management complex. If HR4-6 are an option, it is likely that the decision will be made by health agencies. The ministry of health has an important role in managing the cancer crisis. Cancer must be declared a priority, as had been the case in the fight against the virus. Oncology and surgical societies must coordinate their efforts to facilitate this prioritization. When surgical activities resume, cancers and other life-threatening pathologies will compete with benign pathologies that may be further de- layed. Apart from a ministerial injunction, the aforementioned silo mentality will render the prioritization of lifethreatening pathologies difficult at the local level.

\section{Conclusion}

To prevent excess cancer mortality, it is necessary to anticipate the management of oncology during phase 3 of the pandemic, as it requires a complete reorganization of our healthcare systems.

This adaptation should make it possible to continue cancer management while the virus is still around for an undetermined period of time. The solution will come from our collective intelligence to pool all the resources of our HCS. "Victorious warriors win first and then go to war, while defeated warriors go to war first and then seek to win" (Sun Tzu, The Art of War).

\section{Acknowledgment}

The authors are grateful to Christophe Billard and Remi Heym, Communication Department of the Rouen University Hospital, for their help in creating the figures.

\section{Conflict of Interest Statement}

The authors have no conflict of interests to declare.

\section{Author Contributions}

All of the authors made significant contributions to this paper and have seen and approved the final version of this work. Study concept: A.G., L.S., and J.-J.T. Study design: J.-J.T and L.S. Data acquisition: A.G. and L.S. Quality control of data and algorithms: not applicable. Data analysis and interpretation: J.-J.T., A.G., and L.S. Preparation of this paper: J.-J.T., L.S., A.G., and F.D.F. Editing of this paper: F.D.F., J.-J.T., and L.S. Review of this paper: P.M. and A.B.

\section{References}

1 Grosclaude P, Azria D, Guimbaud R, Thibault S, Daubisse L, Cartron G, et al. COVID-19 impact on the cancer care structuration: example of the multidisciplinary team meeting dedicated to oncology in Occitanie. Bull Cancer. 2020. Available from: https://doi. org/10.1016/j.bulcan.2020.05.001.
2 Lui TK, Leung K, Guo CG, Tsui VW, Wu JT, Leung WK. Impacts of COVID-19 Pandemic on Gastrointestinal Endoscopy Volume and Diagnosis of Gastric and Colorectal Cancers: A Population-based Study. Gastroenterology. 2020 May.

3 European Commission. Estimates of cancer incidence and mortality in 2018. Brussels: European Commission; 2020.
4 Magnani C, Azzolina D, Gallo E, Ferrante D, Gregori D. How Large Was the Mortality Increase Directly and Indirectly Caused by the COVID-19 Epidemic? An Analysis on AllCauses Mortality Data in Italy. Int J Environ Res Public Health. 2020 May; 17(10):E3452. 
5 American College of Surgeons. COVID-19: recommendations for management of elective surgical procedures. 2020. Available from: https://www.facs.org/covid-19/clinical-guidance/elective-surgery.

6 Bartlett DL, Howe JR, Chang G, Crago A, Hogg M, Karakousis G, et al. Management of cancer surgery cases during the COVID-19 pandemic: considerations. Ann Surg Oncol. 2020. doi: 10.1245/s10434-020-08461-2.

7 Bitar N, Kattan J, Kourie HR, Mukherji D, Saghir NE. The Lebanese Society of Medical Oncology (LSMO) statement on the care of patients with cancer during the COVID-19 pandemic. Future Oncol. 2020 Apr;16(11):615-7.

8 Penel N, Bonvalot S, Minard V, Orbach D, Gouin F, Corradini N, et al. French Sarcoma Group proposals for management of sarcoma patients during the COVID-19 outbreak. Ann Oncol. 2020 Jul;31(7):965-6.

9 Simcock R, Thomas TV, Estes C, Filippi AR, Katz MA, Pereira IJ, et al. COVID-19: global radiation oncology's targeted response for pandemic preparedness. Clin Transl Radiat Oncol. 2020 Mar;22:55-68.

10 Tuech JJ, Gangloff A, Di Fiore F, Michel P, Brigand C, Slim K, et al. Strategy for the practice of digestive and oncological surgery during the Covid-19 epidemic. J Visc Surg. 2020 Jun;157(3 3S1):S7-12.

11 ESMO. Cancer Patient Management During the COVID-19 Pandemic. 2020.

12 Dignani MC, Costantini P, Salgueira C, Jordán R, Guerrini G, Valledor A, et al. Pandemic 2009 influenza A (H1N1) virus infection in cancer and hematopoietic stem cell transplant recipients; a multicenter observational study. F1000 Res. 2014 Sep;3:221.

13 Adlhoch C, Gomes Dias J, Bonmarin I, Hubert B, Larrauri A, Oliva Domínguez JA, et al. Determinants of Fatal Outcome in Patients Admitted to Intensive Care Units With Influenza, European Union 2009-2017. Open Forum Infect Dis. 2019 Oct;6(11):ofz462.

14 Liang W, Guan W, Chen R, Wang W, Li J, Xu $\mathrm{K}$, et al. Cancer patients in SARS-CoV-2 infection: a nationwide analysis in China. Lancet Oncol. 2020 Mar;21(3):335-7.

15 Yu J, Ouyang W, Chua MLK, Xie C. SARSCoV-2 transmission in patients with cancer at a tertiary care hospital in Wuhan, China. JAMA Oncol. 2020. Available from: https:// doi.org/10.1101/2020.02.22.20025320.

16 De Felice F, Petrucciani N. Treatment approach in locally advanced rectal cancer dur- ing coronavirus (COVID-19) pandemic: long course or short course? Colorectal Dis. 2020 Jun;22(6):642-3.

17 Mukherjee RK, Back MF, Lu JJ, Shakespeare TP, Wynne CJ. Hiding in the bunker: challenges for a radiation oncology department operating in the Severe Acute Respiratory Syndrome outbreak. Australas Radiol. 2003 Jun;47(2):143-5.

18 CDC CfDCaP. Hydroalcoholic hand gel and strictly observe the relevant CDC recommendation 2020.

19 Centers for Disease Control and Prevention. Infection control recommendation. Atlanta: CDC; 2020

20 Centers for Disease Control and Prevention. Strategies to optimize the supply of PPE and equipment. Atlanta: CDC; 2020.

21 Centers for Disease Control and Prevention. Interim US guidance for risk assessment and public health management of healthcare personnel with potential exposure in a healthcare setting to patients with coronavirus disease (COVID-19). Atlanta: CDC; 2020

22 Ni L, Ye F, Cheng ML, Feng Y, Deng YQ, Zhao H, et al. Detection of SARS-CoV-2-Specific Humoral and Cellular Immunity in COVID-19 Convalescent Individuals. Immunity. 2020 Jun;52(6):971-977.e3.

23 Ota I, Asada Y. The impact of preoperative screening system on head and neck cancer surgery during the COVID-19 pandemic: recommendations from the nationwide survey in Japan. Auris Nasus Larynx. 2020 May. https:// doi.org/10.1016/j.anl.2020.05.006.

24 Hsiao SH, Chen TC, Chien HC, Yang CJ, Chen YH. Body temperature measurement to prevent pandemic COVID-19 in hospitals in Taiwan: repeated measurement is necessary. J Hosp Infect. 2020. Available from: https:// doi.org/10.1016/j.jhin.2020.04.004.

25 European Centre for Disease Prevention and Control. Infection prevention and control and preparedness for COVID-19 in healthcare settings: second update. Solna: ECDC; 2020.

26 Mizumoto K, Kagaya K, Zarebski A, Chowell G. Estimating the asymptomatic proportion of coronavirus disease 2019 (COVID-19) cases on board the Diamond Princess cruise ship: Yokohama, Japan, 2020. Euro Surveill. 2020. Available from: https://doi.org/10.2807/15607917.ES.2020.25.10.2000180

27 Nishiura H, Kobayashi T, Miyama T, Suzuki A, Jung SM, Hayashi K, et al. Estimation of the asymptomatic ratio of novel coronavirus infections (COVID-19). Int J Infect Dis. 2020 May;94:154-5.

28 Ai T, Yang Z, Hou H, Zhan C, Chen C, Lv W, et al. Correlation of chest CT and RT-PCR testing in cor,onavirus disease 2019 (COVID-19) in China: a report of 1014 Cases. Radiology. Forthcoming 2020.

29 Lagier JC, Colson P, Tissot Dupont H, Salomon J, Doudier B, Aubry C, et al. Testing the repatriated for SARS-Cov2: should laboratory-based quarantine replace traditional quarantine? Travel Med Infect Dis. 2020;34:101624.

30 Zhang R, Ouyang H, Fu L, Wang S, Han J, Huang $\mathrm{K}$, et al. CT features of SARS-CoV-2 pneumonia according to clinical presentation: a retrospective analysis of 120 consecutive patients from Wuhan city. Eur Radiol. 2020 Apr.

31 Sirintrapun SJ, Lopez AM. Telemedicine in Cancer Care. Am Soc Clin Oncol Educ Book. 2018 May;38(38):540-5.

32 Tilmans G, Chenevas-Paule Q, Muller X, Breton A, Mohkam K, Ducerf C, et al. Surgical Outcomes after Systematic Preoperative SARS-CoV-2 Screening. Surgery. 2020 May. https://doi.org/10.1016/j.surg.2020.05.006.

33 Adams JG, Walls RM. Supporting the Health Care Workforce During the COVID-19 Global Epidemic. JAMA. 2020 Mar;323(15): 1439.

34 European Centre for Disease Prevention and Control. Rapid risk assessment: outbreak of novel coronavirus disease 2019 (COVID-19): increased transmission globally - fifth update 2020. Solna: ECDC; 2020.

35 Wang S, Xie L, Xu Y, Yu S, Yao B, Xiang D. Sleep disturbances among medical workers during the outbreak of COVID-2019. Occup Med (Lond). 2020 May;2020(May):6.

36 Yin Q, Sun Z, Liu T, Ni X, Deng X, Jia Y, et al. Posttraumatic stress symptoms of health care workers during the corona virus disease 2019. Clin Psychol Psychother. 2020 May;27(3): 384-95.

37 World Health Organization. Coronavirus disease (COVID-19) technical guidance: patient management. Geneva: WHO; 2020.

38 Ngoi N, Lim J, Ow S, Jen WY, Lee M, Teo W, et al.; National University Cancer Institute of Singapore (NCIS) Workflow Team. A segregated-team model to maintain cancer care during the COVID-19 outbreak at an academic center in Singapore. Ann Oncol. 2020 Jul;31(7):840-3.
Treatment of Cancer Patients after the Peak of the COVID-19 Pandemic
Oncology 2020;98:827-835

DOI: $10.1159 / 000509650$ 